\title{
A constitutive model related to the fabric for sand
}

\author{
X. F. Li ${ }^{\text {i) }}$, W. N. Lu ${ }^{\text {ii) }}$ and Z. G. Ma ${ }^{\text {iii) }}$
}

\begin{abstract}
i) Professor, Solid Mechanics Institute, Ningxia University, 489, Helan Mountain Road, Yinchuan city, China.
ii) Ph. D Student, School of Physics and Electronic-electrical Engineering, Ningxia University, 489, Helan Mountain Road, Yinchuan city, China.

iii) Master Student, School of Physics and Electronic-electrical Engineering, Ningxia University, 489, Helan Mountain Road, Yinchuan city, China.
\end{abstract}

\begin{abstract}
In order to better simulated the anisotropy of granular materials, the anisotropic state variable was introduced into the micropolar hypoplastic constitutive model, and the simulation of biaxial compression tests were carried out. The influences of fabric on the width and inclination of the shear band were studied by the FEM and DEM. The following results were found. With the increase of anisotropic state variables, at the same vertical strain, the width and inclination of the shear band remained basically unchanged and the element rotation angle at the same position was increased.
\end{abstract}

Keywords: micropolar, hypoplastic, fabric, anisotropy, shear band

\section{INTRODUCTION}

The geotechnical materials composed by the aggregation of various grains. The materials have obvious anisotropy in physics and have important failure precursors, such as strain localization (He et al, 2017; Shi and Guo, 2018; Vairaktaris et al, 2018). In the hypoplastic constitutive model, micropole theory is introduced to describe the microscopic effects of granular materials. The rotation of particles can be described by micropole theory, which has the advantages of simple and definite parameters (Mindlin, 1964). The fabric tensors can effectively describe the anisotropy of granular materials. Therefore, it had a great theoretical and practical significance that the hypoplastic constitutive model considering fabric was extend to the micropolar form.

Zhiwei Gao and Jidong Zhao (2013) established a plane strain model with fabric by using finite element method (FEM). Sun et al (2013), Fu and Dafalias (2011), Hasan and Alshibli (2012) used the discrete element method (DEM) to analyze the movement of grains in the shear bands. Lin and Wu (2015) respectively used micropolar hypoplastic constitutive model and DEM to simulate simple shear test and biaxial compression test.

In order to applied the micropolar hypoplastic constitutive model with fabric to granular materials, this paper firstly introduced the constitutive correlation function into the hypoplastic constitutive model, and then, extended the hypoplastic constitutive model with fabric to the micropolar.

\section{HYPOPLASTIC CONSTITUTIVE MODEL WITH FABRIC BASED ON MICROPOLAR THEORY}

In order to understand the effect of granular materials fabric on strain localization, an anisotropic hypoplastic constitutive model of micropolar term considering fabric was established. In this model, the relevant parameters of the hypoplastic constitutive and the fabric were obtained by DEM, and then the simulations were calculated by FEM.

\subsection{Micropolar hypoplastic constitutive model with fabric}

In this paper, the anisotropic state variables (A) and Wu-Bauer hypoplastic model (Eq. (1)) were combined to establish the anisotropic hypoplastic constitutive model considering the fabric, and then the model was extended to the form of micropole (Wu et al, 2017). A are described by the fabric tensors (F) and $\boldsymbol{\sigma}$. The transformation from isotropy to anisotropy was realized with the $\mathbf{A}$. The process was as follows:

First, the components of specimen $\mathbf{F}\left(F_{i j}\right)$ and $\mathbf{A}\left(A_{i}\right)$ were calculated (Oda and Nakayama, 1989). The functional relationship between $A_{i}$ and $F_{i j}$ was shown in Eq. (2) (Li and Yuan, 2015).

$$
\stackrel{\circ}{\sigma}=\mathrm{c}_{1} \operatorname{tr}(\boldsymbol{\sigma}) \dot{\boldsymbol{\varepsilon}}+\mathrm{c}_{2} \operatorname{tr}(\dot{\boldsymbol{\varepsilon}}) \boldsymbol{\sigma}+\mathrm{c}_{3} \frac{\operatorname{tr}(\boldsymbol{\sigma} \dot{\boldsymbol{\varepsilon}})}{\operatorname{tr}(\boldsymbol{\sigma})} \boldsymbol{\sigma}+\mathrm{c}_{4}\left(\boldsymbol{\sigma}+\boldsymbol{\sigma}^{*}\right)\|\dot{\boldsymbol{\varepsilon}}\|
$$


where $\stackrel{\sigma}{\sigma}$ is Jaumann stress rate tensor, $c_{i}(i=1,2,3,4)$ is a dimensionless parameter, $\boldsymbol{\sigma}$ is the stress tensor, $\dot{\boldsymbol{\varepsilon}}$ is strain rate tensor, $\boldsymbol{\sigma}^{*}$ is the deviatoric stress tensor, $\boldsymbol{\sigma}^{*}=\boldsymbol{\sigma}-\operatorname{tr}(\boldsymbol{\sigma}) I / 3$.

$$
A=\frac{\sigma_{i j}}{\sigma_{\mathrm{m}}} F_{i j}-A_{0}
$$

where $\sigma_{i j}$ is the component of the $\boldsymbol{\sigma}, \sigma_{\mathrm{m}}$ is the component of the $\boldsymbol{\sigma}^{*}, A_{0}$ is the datum point (the initial anisotropy state variable), the stress state at this point is consistent with the stress state when the specimen parameters were determined.

Then, by combining physical quantities such as porosity, material parameters of the critical state line equation ( $f(e)$ ) and $\boldsymbol{A}$ with Eq. (1). The anisotropic hypoplastic constitutive model (Eq. (3)) considering the fabric was established. On the basis of Bauer critical state line (Bauer, 1995), Eq. (4) introduced the fabric related terms ( $\mathrm{Li}$ and Yuan, 2015).

$$
\begin{gathered}
\stackrel{\circ}{\boldsymbol{\sigma}}=\mathrm{c}_{1} \operatorname{tr}(\boldsymbol{\sigma}) \dot{\boldsymbol{\varepsilon}}+\mathrm{c}_{2} \operatorname{tr}(\dot{\boldsymbol{\varepsilon}}) \boldsymbol{\sigma}+\mathrm{c}_{3} \frac{\operatorname{tr}(\boldsymbol{\sigma} \dot{\boldsymbol{\varepsilon}})}{\operatorname{tr}(\boldsymbol{\sigma})} \boldsymbol{\sigma}+\mathrm{c}_{4} f(e)\left(\boldsymbol{\sigma}+\boldsymbol{\sigma}^{*}\right)\|\dot{\boldsymbol{\varepsilon}}\| \\
f(e)=\left(\frac{e-e_{\min }}{e_{\Gamma}-\lambda_{\mathrm{c}}\left(P / P_{\mathrm{a}}\right)^{\zeta}+A-e_{\text {min }}}\right)^{a}
\end{gathered}
$$

where $e$ is the porosity, $e_{\min }$ is the minimum porosity, $e_{\Gamma}, \lambda_{\mathrm{c}}$ and $\zeta$ are the material parameters that determine the critical state line, $a$ can be measured by experiments (Wu and Kolymbas, 2000), $e_{\Gamma}, \lambda_{\mathrm{c}}, \zeta$ and

$$
\begin{aligned}
& \stackrel{\circ}{\boldsymbol{\sigma}}=\mathrm{c}_{1} \operatorname{tr}(\boldsymbol{\sigma}) \dot{\boldsymbol{\varepsilon}}+\mathrm{c}_{2} \operatorname{tr}(\dot{\boldsymbol{\varepsilon}}) \boldsymbol{\sigma}+\mathrm{c}_{3} \frac{\operatorname{tr}(\boldsymbol{\sigma} \dot{\boldsymbol{\varepsilon}})-\operatorname{tr}(\mathbf{M K})}{\operatorname{tr}(\boldsymbol{\sigma})} \boldsymbol{\sigma}+\mathrm{c}_{4} f(e) \sqrt{\|\dot{\boldsymbol{\varepsilon}}\|^{2}+l^{2}\|\mathbf{K}\|^{2}}\left(\boldsymbol{\sigma}+\boldsymbol{\sigma}^{*}\right) \\
& \stackrel{\circ}{\mathbf{M}}=\mathrm{c}_{1} \operatorname{ltr}(\boldsymbol{\sigma}) \mathbf{K}+\mathrm{c}_{2} \frac{\operatorname{tr}(\dot{\boldsymbol{\varepsilon}})}{l} \mathbf{M}+\mathrm{c}_{3} \frac{\operatorname{tr}(\boldsymbol{\sigma} \dot{\boldsymbol{\varepsilon}})-\operatorname{tr}(\mathbf{M K})}{l \operatorname{tr}(\boldsymbol{\sigma})} \mathbf{M}+2 \mathrm{c}_{4} f(e) \sqrt{\|\dot{\boldsymbol{\varepsilon}}\|^{2}+l^{2}\|\mathbf{K}\|^{2}} \frac{\mathbf{M}}{l}
\end{aligned}
$$

\subsection{FEM stiffness matrix}

The FEM stiffness matrix was obtained by partial differentiation for Eq. (5) and (6). The FEM stiffness matrix was $7 \times 7$ matrix, that was, $\mathbf{S}=\mathbf{H}(\boldsymbol{\sigma}, \dot{\boldsymbol{\varepsilon}}, \mathbf{M}, \mathbf{K})$ could be expressed as Eq. (7).

By the time step $\Delta t$, the next stress state could be expressed as Eq. (8).

$$
\begin{gathered}
\mathbf{S}=\left[\begin{array}{ll}
\frac{\partial \stackrel{\sigma}{i j}_{i j}}{\partial \dot{\boldsymbol{\varepsilon}}_{m n}}(5 \times 5) & \frac{\partial \stackrel{\circ}{\mathbf{\sigma}}_{i j}}{\partial \mathbf{K}_{m n}}(5 \times 2) \\
\frac{\partial \dot{\mathbf{M}}_{i j}}{\partial \dot{\boldsymbol{\varepsilon}}_{m n}}(2 \times 5) & \frac{\partial \stackrel{\circ}{\mathbf{M}}_{i j}}{\partial \mathbf{K}_{m n}}(2 \times 2)
\end{array}\right] \\
\boldsymbol{\sigma}_{t_{n+1}}=\boldsymbol{\sigma}_{t_{n}}+\mathbf{H}(\boldsymbol{\sigma}, \dot{\boldsymbol{\varepsilon}}, \mathbf{M}, \mathbf{K}) \Delta t
\end{gathered}
$$

where $\boldsymbol{\sigma}_{t_{n+1}}$ is the stress tensor of the next time step, $\boldsymbol{\sigma}_{t_{n}}$ is the stress tensor of the current time step.

\subsection{Calculation model}

In this paper, two plane strain biaxial compression test models were established. The DEM models height $(H)$ and the width $(W)$ were $70 \mathrm{~mm}$ and $35 \mathrm{~mm}$ respectively (Fig. 1(a)). The FEM models $H$ and $W$ were $48 \mathrm{~mm}$ and $16 \mathrm{~mm}$ respectively (Fig. 1(b)). The relevant parameters of the DEM models and FEM models were shown in table 1 and 2.

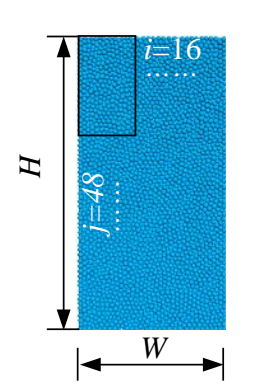

(a)

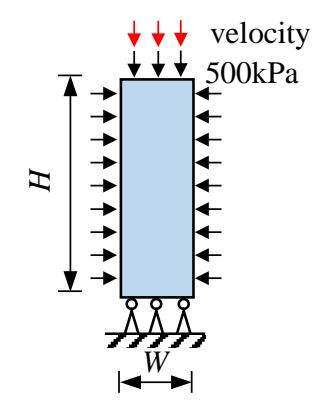

(b)
Fig. 1. The two calculation models and corresponding relationship.

\section{THE NUMERICAL SIMULATION}


Table 1 The relevant parameters of the DEM model.

\begin{tabular}{lllllll}
\hline $\begin{array}{l}\text { density/ } \rho \\
\left(\mathrm{kg} / \mathrm{m}^{3}\right)\end{array}$ & $\begin{array}{l}\text { modulus of } \\
\text { elasticity/ } E \\
(\mathrm{MPa})\end{array}$ & $\begin{array}{l}\text { normal contact } \\
\text { stiffness/ } K_{\mathrm{n}} \\
(\mathrm{N} / \mathrm{m})\end{array}$ & $\begin{array}{l}\text { granular normal to } \\
\text { tangential stiffness } \\
\text { ratio/ }\left(K_{\mathrm{n}} / K_{\mathrm{s}}\right)\end{array}$ & $\begin{array}{l}\text { damping } \\
\text { coefficient }\end{array}$ & $\begin{array}{l}\text { coefficient of } \\
\text { friction between } \\
\text { grains } / f_{\mathrm{g}}\end{array}$ & $\begin{array}{l}\text { coefficient of } \\
\text { friction between } \\
\text { grain and wall } / f_{\mathrm{w}}\end{array}$ \\
\hline 1600 & 20 & $1 \times 10^{8}$ & 1.00 & 0.7 & 0.4 & 0 \\
\hline
\end{tabular}

Table 2 The relevant parameters of the FEM model.

\begin{tabular}{lllllll}
\hline$E /(\mathrm{MPa})$ & poisson ratio $/ \mu$ & friction angle $/ \varphi\left(^{\circ}\right)$ & $\mathrm{C}_{1}$ & $\mathrm{C}_{2}$ & $\mathrm{C}_{3}$ & $\mathrm{C}_{4}$ \\
\hline 20 & 0.25 & 45 & -53.33 & -234.55 & -210.29 & -105.90 \\
\hline
\end{tabular}

\subsection{Calculation results}

Fig. 2 shown the distribution of $\alpha$ in schemes $1 \sim 4$ at different $\varepsilon_{\mathrm{v}}$. Fig. 3 shown the evolution of the average stress ratio $n$ (the average the maximum principal stress $\left(\sigma_{1}\right)$ over the mean minimum principal stress $\left(\sigma_{3}\right)$ at the top of the FEM model with the $\varepsilon_{\mathrm{V}}$ in schemes $1 \sim 4$. Fig. 4 shown the evolution of rotation angle $\left(\alpha_{\mathrm{m}}\right)$ of monitoring points with the $\varepsilon_{\mathrm{v}}$ in schemes $1 \sim 4$. The monitoring points in schemes $1 \sim 4$ were the same, and

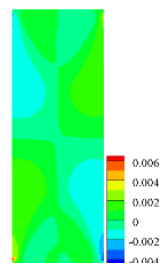

(a) $\varepsilon_{\mathrm{V}}=2.4 \%$ $\varepsilon_{\mathrm{V}}=4.4 \%$

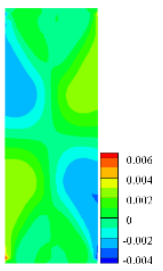

(b) $\varepsilon_{\mathrm{V}}=2.8 \%$

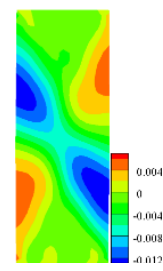

(c) $\varepsilon_{\mathrm{V}}=3.5 \%$

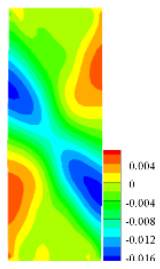

(d) $\varepsilon_{\mathrm{v}}=3.6 \%$
Fig. 2. The distribution of $\alpha$ in schemes $1 \sim 4$.

Fig. 3 shown that, the curves of $n-\varepsilon_{\mathrm{V}}$ for schemes $1 \sim$ 4 could be divided into three stages: elastic stage, hardening stage and softening stage.

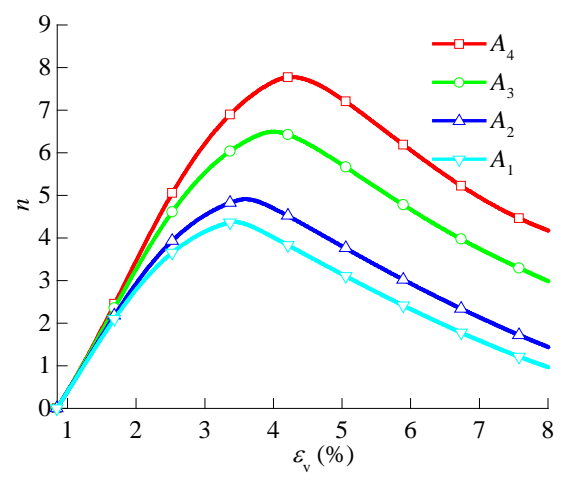

Fig. 3. The curves of $n-\varepsilon v$.

In the hardening stage, with the increase of $A_{i}$, the peak values of the curves of $n-\varepsilon_{\mathrm{v}}$ increased in schemes $1 \sim 4$. In the softening stage, with the increase of $A_{i}$, the evolution of the curves of $n-\varepsilon_{\mathrm{V}}$ in schemes $1 \sim 4$ was basically the same, and the slopes after the peak of the curves of $n-\varepsilon_{\mathrm{v}}$ were basically the same. The reason was the fact that shear band has been formed and the FEM model was fully softened. the monitoring points position are the element with the maximum shear stress in scheme 1 when $\varepsilon_{\mathrm{v}}=2.8 \%$.

Fig. 2 shown that, in terms of width $\left(w_{\mathrm{s}}\right)$ and inclination of the shear band $(\theta)$, at the same $\varepsilon_{\mathrm{v}}$, with the increase of $A_{i}, \theta$ and $w_{\mathrm{s}}$ were basically unchanged. For example, when $\varepsilon_{\mathrm{V}}=4.4 \%$, the $\theta$ is about $50^{\circ}$, and $w_{\mathrm{s}}$ was about $9 \mathrm{~mm}$ in schemes $1 \sim 4$ (Fig. 2(e) (h)). In terms of the dilatation phenomenon of the FEM model, with the increase of $A_{i}, \theta$ and $w_{\mathrm{s}}$ were basically unchanged.

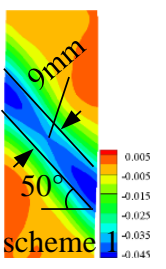

(e) $\varepsilon_{\mathrm{V}}=4.4 \%$

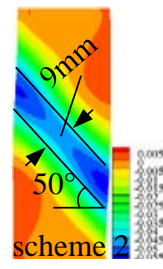

(f) $\varepsilon_{\mathrm{V}}=4.4 \%$

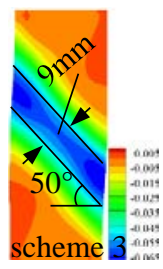

(g) $\varepsilon_{\mathrm{V}}=4.4 \%$

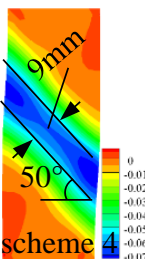

(h)
Fig. 4 shown that, the curves of $\alpha_{\mathrm{m}}-\varepsilon_{\mathrm{v}}$ of FEM model for schemes $1 \sim 4$ could be divided into three stages: approximate 0 stage, nonlinear stage and linear stage. In the nonlinear stage, with the increase of $A_{i}$, the $\alpha_{\mathrm{m}}$ of schemes $1 \sim 4$ increased at the same $\varepsilon_{\mathrm{v}}$. This indicates that the higher degree of anisotropy had the faster relative motion speed of the hanger and dropped side of the shear band in the process of forming shear band.

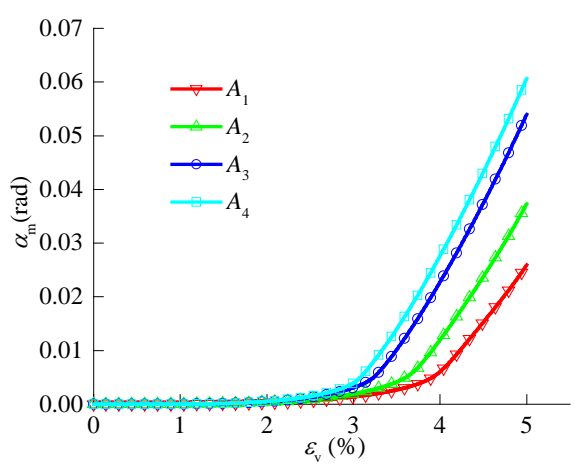

Fig. 4. The curves of $\alpha_{\mathrm{m}}-\varepsilon_{\mathrm{v}}$.

\section{CONCLUSIONS}

The FEM simulation of the biaxial compression 
plane strain models were carried out, and the following conclusions were obtained:

(1) In order to better simulated the anisotropy of granular materials, the correlation function of fabric was added into the micropolar hypoplastic constitutive model, and the micropolar hypoplastic constitutive model considering the fabric was established.

(2) The curves of average stress ratio of the upper end of the FEM model vertical strain can be divided into three stages: elastic stage, hardening stage and softening stage.

(3) With the increase of anisotropic state variables, at the same vertical strain, the width and inclination of the shear band remained basically unchanged and the rotation angle at the same position increased.

\section{ACKNOWLEDGMENTS}

This work was financially supported by the National Key R\&D Program of China (2017YFC0504404), the Key R\&D Program of Ningxia Hui Autonomous Region Projects of International Cooperation and Exchanges (2018DWHZ0084) and the National Natural Science Foundation of China (NO: 51669027) and these supports are gratefully acknowledged.

\section{REFERENCES}

1) Bauer E. (1995): Constitutive modelling of critical states in hypoplasticity. In Pande and Pietruszczak (eds). Numerical models in geomechanics-NUMOG V, Balkema, 15-20.

2) Fu, P. and Dafalias, Y. F. (2011): Fabric evolution within shear bands of granular materials and its relation to critical state theory. International Journal for Numerical \& Analytical Methods in Geomechanics, 35(18), 1918-1948.

3) Gao, Z. and Zhao, J. (2013): Strain localization and fabric evolution in sand. International Journal of Solids and Structures, 50(22-23), 3634-3648.

4) Hasan, A. and Alshibli, K. (2012): Three dimensional fabric evolution of sheared sand. Granular Matter, 14(4), 469-482.

5) He, X., Cai, G. Zhao, C. and Sheng, D. (2017): On the stressforce-fabric equation in triaxial compressions: some insights into the triaxial strength. Comput. Geotech, 85, 71-83.

6) Li, X., Yuan, Q. and Wang, X. (2015): A critical state anisotropic model with state-dependent of sand. Journal of Civil, Architectural \& Environmental Engineering, 37(3), 70-78 (in Chinese).

7) Lin, J. and Wu, W. (2015): A comparative study between dem and micropolar hypoplasticity. Powder Technology, 293, 121-129.

8) Mindlin RD. (1964): Microstructure in linear elasticity. Arch Rational Mech. Anal., 16, 51-78.

9) Oda, M. and Nakayama, H. (1989): Yield function for soil with anisotropic fabric. Journal of Engineering Mechanics, 115(1), 89-104.

10) Shi, J. and Guo, P. (2018): Fabric evolution of granular materials along imposed stress paths. Acta Geotechnica, 13(6), 1341-1354.

11) Sun, W., Kuhn, M. R. and Rudnicki, J. W. (2013): A multiscale DEM-LBM analysis on permeability evolutions inside a dilatant shear band. Acta Geotechnica, 8(5), 465-480.

12) Vairaktaris, E. and Theocharis, A. I., Dafalias, Y. F. (2018): Correlation of fabric tensors for granular materials using 2D DEM. Acta Geotechnica (3), https://doi.org/10.1007/s1
1440-018-0755-1.

13) Wu, W. and Kolymbas, D. (2000): Hypoplasticity then and now. Constitutive Modelling of Granular Materials Heidelberg, Berlin, 57-105.

14) Wu, W., Lin, J. and Wang, X. (2017): A basic hypoplastic constitutive model for sand. Acta Geotechnica, 12(6), 13731382. 